\section{CASES OF INTESTINAL OBSTRUCTION TREATED BY LAPAROTOMY. ${ }^{1}$}

BY THOMAS JONES, M.B.LoND., F R.C.S.ENG., \&e, SURGEON TO THE MANCIIESTER ROYAL INFIRMARY.

CASE 1. Intestinal obstruction; adhesions around the vermiform appendix ; laparotomy, with removal of appendix and liberation of cacum; recovery.-Mr. G- aged thirty, seen with Dr. Rhodes on Feb. 20th, 1889. The patient had been ill for two days, suffering from pain referred to the cæcal region, vomiting, some abdominal distension, and no action of the bowels. A history of an acute attack of peritonitis thirteen years previously was obtained. Since then, with the exception of milder attacks of pain on several occasions, he has been in the enjoyment of good health, and is a very active athlete. For several months prior to the present illness he had been troubled with constipation, with an increasing difficulty in obtaining an action of the bowels. Has occasionally had colicky pains over the cæunm. The usual treatment was resorted to-enemata, rectal feeding, and opium to relieve the pain. Some relief was obtained by washing out the stomach. The symptoms of obstruction, however, continued, and assumed a more aggravated form. Stercoraceous vomiting appeared about the tenth day. Laparotomy was performed on March 2nd, the thirteenth day from the beginning of the attack. Incision made in the right linea semilunaris. Directly the abdomen was opened ex tensive firm adhesions, due no doubt to the old attack of peritonitis, were found in the creal region. The ileum was attached to the cæcum, and the latter fixed down firmly. The appendix vermiformis was jammed down in the pelvis, where it was held by strong b unds. These were separated, the appendix brought up into the wound, a ligature placed round it near the cæcum, and some three inches of it removed; its lumen being completely obliterated, no special precautions were taken to turn and stitch its peritoneal covering. After careful cleansing the abdomen was closed. The after-history of this case is very simple. The bowels acted freely the same day some eight hours after the operation. The symptoms of obstruction were immediately and completely relieved, and the wound gave no trouble. Now, after the lapse of twenty-one months, Mr. Gexpresses himself as being in the enjoyment of excellent health. He has resumed his athletic exercises, and is able to ride a bicycle with ease and comfort. He is careful with his eating and drinking, and althoagh his bowels are still somewhat sluggish, a regular daily action is generally secured.

CASE 2. Intestinal obstruction; knuckle of intestine found adherent to the mouth of an old femoral hernia; laparotomy; recovery._Elizabeth $\mathrm{G}$ the infirmary on July 25th, 1890. For three months she has oceasionally had attacks of romiting, accompanied with severe pains in the abdomen. The attacks have come on very suddenly, usually lasting about half an hour. The last attack commenced a week prior to her admission, with severe pain in the lower part of the abdomen. Vomiting set in almost at once, and became stercoraceous six days later, the day before she entered the infirmary. Constipation was absolute, but there never was complete obstruction, as flatus was passed up to the time of operation. Her condition when admitted was very serious; the eyes were sunken, and the vomiting was almost incessant; her state reminded one very forcibly of a patient suffering from strangulated hernia. At this time she was on the medical side, where belladonna was freely administered, and the vomiting checked for eighteen hours. Enemata were also given, bringing away some scybala. When seen a few days after admission, her state was most critical ; the abdomen was very distended, and every where tympanitic. A swelling the size of a walnut was discovered in the right femoral region. This the patient stated had existed for years, and caused no inconvenience. It was dull on percussion, and gave no impulse on coughing. It was thought that possibly a piece of bowel had become strangulated at the upper part of the femoral canal; but as the local symptoms referable to this region were so indistinct, it was thought advisable

I A communication made to the Clinical Society of Manchester on Dec. 16th, 1890 . to open the abdomen in the median line. This was done while the patient was under the influence of chloroform. As soon as the abdominal cavity was opened the fingers were introduced and passed down in the direction of the right femoral region. Here a small knuckle of small in. testice was readily found adherent in about half its circum. ference. The adhesions were carefully separated, and the portion of gut so detached brought up into the wound and examined. There was some oozing from the surface which had been attached; otherwise it was healthy, so it was re. turned, and the wound in the abdominal wall completely closed, the paritoneum being separately sutured with catgut. The wound was dressed with carbolic ganze and wood wool pads. About three hours after the operation the patient passed a very copious motion. At first alimentation was effected by the rectum, fluid food being administered by the mouth on the third day. The wound was not dressed for a week, when it was found practically healed. Twentyfive days after the operation she left the infirmary, wearing a truss and an abdominal belt.

CASE 3. Intestinal obstruction; laparotomy; death.J. W- aged thirty-eight, seen in consultation with Dr. Edward of Riwtenstall and Dr. Stewart of Bacup on April 23rd, 1890. The following history was obtained. The patient has suffered for years from a right oblique inguinal hernia, and has worn a truss. The rupture has not been down for a long time, and never when he has the truss on. On April 17th he felt great uneasiness in the right inguinal region, which became worse towards evening, when he was sick once or twice. He retired to bed early, but had to get up again, owing to the increasing discomfort. After being up and moving about for an hour or so, he went to bed again, but felt very uneasy all night. The next day he remained the same, employed some domestic remedies, but obtained no relief. Sickness now became persistent, and remained so until the end; it was attended with distressing and almost constant hiccough. Enemata, repeatedly tried, brought little or no fæcal matter away. A couple of pints of fluid could be easily thrown up. When I saw him he was lying propped up in bed, and had the facial expression of one suffering from obstruction. Tongue dry in centre; pulse 90 , small and inclined to be thready; no fever. He was violently sick while he was being examined. Abdomen pretty uniformly distended and every where resonant; no tenderness, not even in the vicinity of the hernial sac, which was empty, and the finger could be easily passed up the inguinal canal. Diagnosis: Intestinal obstruction, probably connected with the old hernia, possibly adhesions about the neck. The condition of the patient was most un. favourable, still we deemed it our duty to give him the only possible chance by immediate operation. Abdominal section was therefore performed by a median incision. On opening the abdomen distended small intestine protruded, and on passing the left forefinger towards the right inguinal region some coils were found heaped up. On examining more carefully an opening was found, the anterior edge of which was exceedingly sharp, and attached to it was a loop of small intestine. The opening corresponded with the internal ring, and we concluded that the gut bad become strangulated immediately on its entrance into the canal. I attempted to pull up the coil of intestine, but feared to use much force lest the bowel should give way. Any attempt to divide the stricture from the inside would be too hazardous, so I decided to cut down on the sac. This was not an easy matter, as it was empty, and the light very indifferent. However, I succeeded in opening it, and found that it contained a very small quantity of clear serum. Now, by fixing the internal ring and dilating it somewhat with the finger in the abdominal cavity, and pressing the coil with the fingers in the sac, I reduced it after a considerable amount of trouble. The internal ring was so movable that it, together with the knuckle of intestine strangulated within it, could be pulled up a considerable distance into the abdominal cavity. After reducing the gut it was examined, and found to be deeply congested, but free from any deposit of lymph. The sac was isolated from the neighbouring structures, transfixed with carbolised silk, tied in a Staffordshire knot, and the portion below the ligature removed. This wound was cleaned with a perchloride solution, dusted over with boracic powder, and closed entirely with a continuous silk suture. The abdominal wound was also closed with four silk sutures passed through skin and parietal peritoneum on each side, and three superficial silk sutures. Unfortunately, the shock of 
the operation proved too much for the patient in his then exhausted condition; he was very collapsed, and did not respond to the medicaments employed. He died about three hours afterwards.

(To be concluded.)

\section{F I B R O I P N E U M O I A.}

\section{By A. G. AULD, M.D.}

(Concluded from page 1310.)

I Now submit the following cases of primary fibroid pneumonia, the clinical notes of which I have obtained through the kindness of Drs. Finlayson and Coats, under whose care they respectively were.

CASE 1.1-Alex. C___, aged forty, grocer, enjoyed good health till three weeks before admission to Glasgow Western Infirmary. Illness began with a chill, which gradually became worse, with cough and expectoration. Examination revealed signs of consolidation at the right apex and râles at the bases of both lungs. Morning temperature $101 \cdot 2^{\circ}$; evening, $103^{\circ}$; falling to $101^{\circ}$ on the evening of the third day. After the fifth day it fell to $99.4^{\circ}$ in the morning and $100^{\circ}$ in the evening, and averaged this rate till death. Respiration 26; pulse 120 . After a few days there were signs of extensive consolidation in both lungs. The expectoration was greenish-yellow and considerably abundant; the urine was free from albumen. The diagnosis was acute phthisis. The patient died three weeks after admission, making in all six weeks in which the disease ran its course. Dr. Coats conducted the post-mortem examination. It was therein found that both lungs in their lower two-thirds were consolidated. The consolidation was generally red, and somewhat resembled ordinary hepatisation, the cut surface being granular, but the lower lobe of the left lung was smooth on section and of a slate grey tint, and this lung was somewhat shrunken. The right lung was of normal bulk. Both apices were crepitant. The bronchi were throughout filled with thick yellow pus, and in the lower part of the left lung were generally somewhat dilated; elsewhere of normal calibre. Not a single tubercle was anywhere to be found. The other organs were normal Afrer hardening pieces from all parts of both lungs in Müller's fluid, and afcerwards in alcohol, I mounted and examined forty sections. The left base contained areas of dense connective tissue, formed by the fusion of intra-alveolar masses with the fibrous walls of the alveoli. The remaining condensation in both lungs had the following characters. In some areas the alveoli were filled with fibrin and large epithelial cells. Occasionally the fibrin was replaced by a gelatinous exudation like frogs' spawn. Encroaching on these areas, and occupying much the greater extent of the consolidated parts, was the characteristic induration of the intra-alveolar products-globular pedunculated masses of fibrous tissue, very vascular, and showing frequent extravasations (see illustration). The alveolar walls were also sumewhat thickened by the nuclear growth. White and red blood cells and proliferated epithelium in exceeding abundance occupied such space of the alveoli as was unoccupied by the fibrous tissue, and side by side with the tissue containing alveoli were not a few which were exclusively occupied by the former products. Neither the pleura nor interlobular septa showed any thickening, but the sheaths of the smaller bloodvessels and bronchioles participated in the new growth. The bronchial mucous membrane was crammed with lymph cells and its surface stripped of its epithelial layer, but at rare intervals a few ciliated columnar cells derived from this source were to be found in an alveolar cavity. A drop of the bronchial pus was examined by Dr. R. Buchanan and found to contain the capsulated diplococcus pneumoniæ of Fränkel. Both the muscular and inner coats of the pulmonary artery were thickened.

CASE 2.-John M- aged sixty-three, shoemaker, began to suffer six months before admission to hospital from a congh and difficulty of breathing and swelling of the feet and legs. He had been asthmatic for some years. Three weeks ago the chest symptoms got so bad that he was forced to remain in bed. He was emaciated and sallow; temperature normal, and occasionally subnormal; pulse 98 ; respiration 32. Cough slight, and attended by little or no ex-

1 Only a brief summary on the clinical side can be given here. pectoration. Mucous I Âles in both lungs. Great dyspnoea, which amounted at times to orthopnoa. Death took place two days after admission. Post-mortem examination : The left lung was adherent at the extreme apex. It was highly cedematous throughout, with partial condensation, while at the extreme apex and beneath the adhesions there was a darkly pigmented cicatrix, with cretaceous matter in the midst. The right lung was more extensively adherent; it also contained cicatrices with cretaceous matter, and a small ragged cavity. There was no appearance of active or recent tubercular condensation. Otherwise this lung was odematous and partly condensed. The heart was found to be considerably enlarged, and the muscular walls at the extreme apex of both ventricles were very thin. There was exteusive atheromatous disease of the arteries. The kidneys were granular, and the surface of the liver highly granular. The condensation of the lungs was chiefly of the lower lobes. It was red on section and sljghtly granular, and had not caused any shrinking; there was no bronchiectasis. Microscopically, it presented features in every respect similar to those described in the first case. The fibrous masses in the alveoli, however, were herein more dense and homogeneous, resembling the sclerosed glomeruli of contracted kidney. Large swollen epithelial cells, often bi-nucleated, were in considerable abundance. Many of the alveoli also contained a fibrino-hæmorrhagic exudation. Their walls could hardly be said to be at all thickened, and the septa were in a like condition. The bronchial mucous membrane was perfectly normal, but some columnar epithelium was in this case also to be found in a few of the air.cells, although it is just possible that this was brought about in the cutting of the sections. A very marked feature, and one of considerable interest from another point of view, was the extreme atrophy of the muscular coat of the bronchial and pulmonary arteries. Sometimes the muscle existed only as a series of dots along the walls of the smaller vessels, in the midst of the fibroid tissue, and was well brought out in mahogany-red by picro-carmine in a light.pink ground. ${ }^{2}$

The first of these cases is very striking, and in some respects unique. The second was associated with fibroid changes in other organs; it is evident, however, that the pulmonary consolidation was recent.

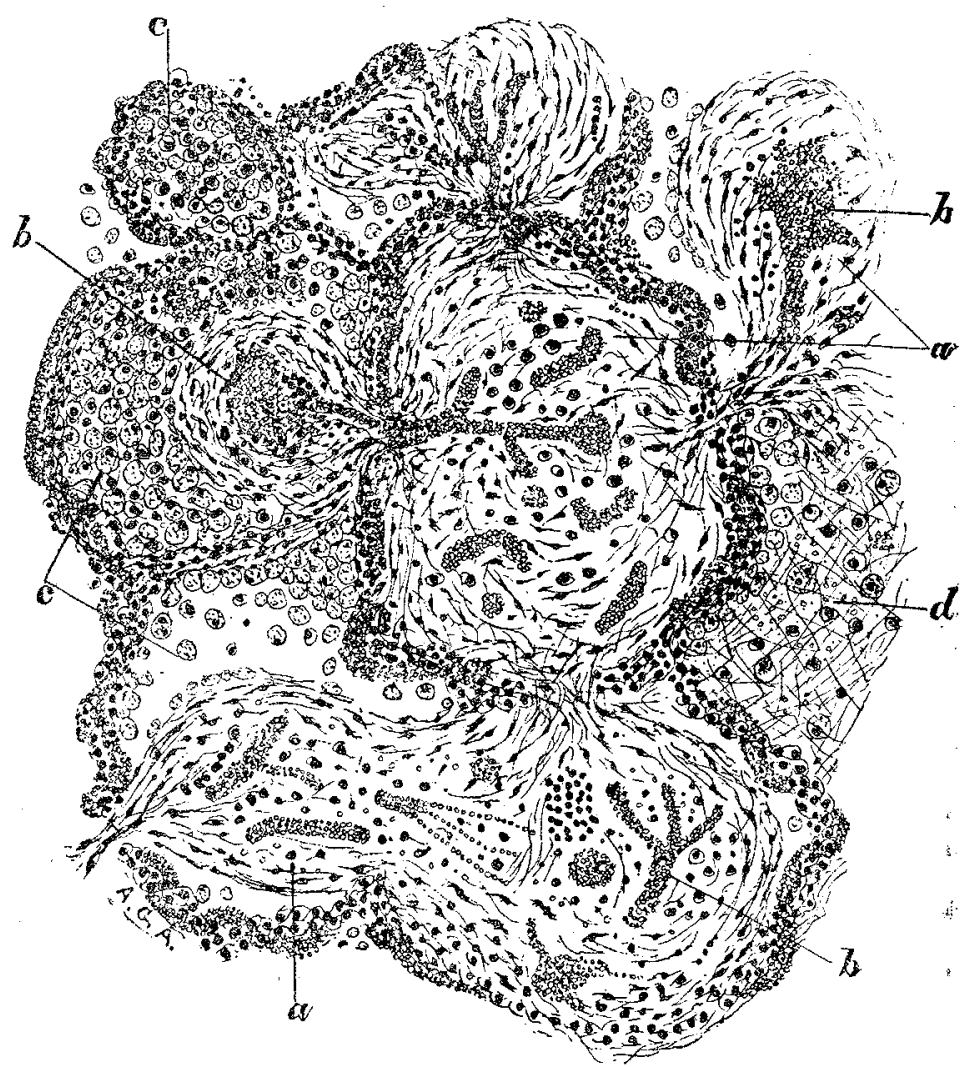

Fibroid Pneumonia.

$a$, Masses of connective tissue developed within the alveoli in some places fusion with the alveolar walls has taken in som place. considerable extravasation is seen. $c$, Desquamated and fatty epithelium mingled with some leucocytes and red $\times 120$, considerably reduced.

2 This phenomenon conclusively shows that the observations of Gul and Sutton on the muscular coat of the arteries in Bright's disease were in some cases, at any rate, perfectly correct; but which some have been unwilling to concede. 